\title{
Subtle Attention Guidance for Real Walking in Virtual Environments
}

\section{Conference Paper}

Author(s):

Nonino, Emanuele; Gisler, Joy; Holzwarth, Valentin; Hirt, Christian; Kunz, Andreas (D)

Publication date:

2021

Permanent link:

https://doi.org/10.3929/ethz-b-000509754

Rights / license:

In Copyright - Non-Commercial Use Permitted

Originally published in:

https://doi.org/10.1109/ISMAR-Adjunct54149.2021.00070 


\section{Subtle Attention Guidance for Real Walking in Virtual Environments}

\author{
Emanuele Nonino * \\ ETH Zurich
}

\author{
Joy Gisler ${ }^{\dagger}$ \\ ETH Zurich
}

\author{
Valentin Holzwarth \\ University of Liechtenstein
}

\author{
Christian Hirt\$ \\ ETH Zurich
}

\author{
Andreas Kunz \\ ETH Zurich
}

\begin{abstract}
Virtual reality is today being applied to an increasing number of fields such as education, industry, medicine, or gaming. Attention guidance methods are used in virtual reality to help users navigate the virtual environment without being overwhelmed by the overabundance of sensory stimuli. However, visual attention guidance methods can be overt, distracting and confusing as they often consist of artefacts placed in the center of the user's field of view. This is the case for the arrow method, which consists of an arrow pointing towards a target object and which serves as a reference for our study. In this paper, we compare such an arrow to two methods that are less distracting and more subtle: haptic feedback and temporal luminance modulation. The haptic feedback method guides a user to a target using controller vibration. The temporal luminance modulation method makes use of flickering visual artefacts placed at the user's peripheral field of view and thus do not cover regions of interest that are typically in the central field of view. This creates a subtle attention guidance since these flickering artefacts can be perceived by the user, but not recognized in terms of form and shape. To compare the different attention guidance methods, we designed a virtual environment that can be explored through real walking, wherein a user performs a search task. We then conducted a pilot study with seven participants to compare the haptic feedback and the temporal luminance modulation methods to the arrow method and to a baseline condition of navigation without any attention guidance. The preliminary results suggest that all three methods are more effective than the condition without guidance. Moreover, the temporal luminance modulation method appears to be comparable to the more effective, but non-subtle arrow method in terms of task completion time.
\end{abstract}

Keywords: Virtual reality, attention guidance, virtual environments

Index Terms: Human-centered computing-Human computer interaction (HCI) - Interaction paradigms - Virtual reality; Humancentered computing - Visualization-Empirical studies in visualization

\section{INTRODUCTION}

Today, Virtual Reality (VR) is applied to many different fields such as education, industry, medicine, or gaming. In many of these applications, users can navigate freely and are required to focus their attention on specific portions of the Virtual Environment (VE). Other than movies, VEs allow users to explore and interact with their surrounding virtual space. However, problems arise if users get lost in such a large VE. To avoid this, it is important that users receive help, otherwise visual key elements for a specific VR application could be missed. However, this requires guiding users through a VE without distracting them from the given task. In some VR applications,

\footnotetext{
*e-mail: enonino@student.ethz.ch

†e-mail: gisler@iwf.mavt.ethz.ch

†e-mail: valentin.holzwarth@uni.li

§e-mail: hirtc@iwf.mavt.ethz.ch

Ie-mail: kunz@iwf.mavt.ethz.ch
}

guiding users' attention to relevant places in the VE could increase the amount of relevant information that can be retrieved from the VE. For example, in VR assembly tasks, the task completion time is influenced by the time required to reach and find the individual components that need to be assembled [6]. Consequently, guiding users' attention towards a target object could reduce the duration of the teaching sequences, leading to an increased training efficiency.

Attention Guidance (AG) consists of shifting the attention of a user towards a target object [3]. In particular, AG is used if a target object is located in the peripheral part or outside the user's Field of View (FOV). To guide users, the AG methods use stimuli conveyed through different sensory modalities. AG methods predominantly rely on the visual, auditory, and tactile sensory channels $[2,3,13,14$, $18,19,22]$. The effectiveness of an AG method is often related to the completion time required for the user to reach and find a target object in the VE. However, an ideal AG method should also avoid using stimuli that occupy too much of the user's mental capacity, as this could affect their performance in the VR task at hand. Thus, the concept of subtlety is used to distinguish subtle AG methods that do not distract the user from overt AG methods [3]. While overt methods are typically superior to subtle methods, they might occupy too much of other important visual information in the VE. For example, in case of redirected walking techniques, the so-called "resets", which show an arrow when a user is supposed to turn on spot, cannot be combined well with AG techniques like e.g. an attention funnel [17] for a Mixed Reality (MR) application. Further, they might cover alerts like the "Chaperone" grid of today's VR systems. To compare these AG methods, specific VEs are designed, which are usually referred to as controlled VEs. In a controlled VE, specific tasks (e.g. a search task or an assembly task) are carried out by the users. Controlled VEs can be classified depending on whether the users are stationary or can navigate the VE by real walking. In such VEs, where real walking is not allowed (i.e. stationary), users can only rotate their head or body to determine the visible section of the VE. This is the case for cinematic VE in which users watch a $360^{\circ}$ video using a Head-mounted Display (HMD) or other VR devices. In a real walking enabled VE, users are also able to navigate the scene and thus have more complex interactions with the virtual objects in the environment. Various metrics are then used to assess the AG methods' performance, such as search time or heat-maps of the viewing direction.

In this paper, we present the following contributions:

- Design and implementation of a controlled VE, based on a search task that gives users the possibility to actively explore the virtual space through real walking.

- Comparative pilot study between AG methods addressing different sensory channels within a real walking enabled VE.

\section{Related Work}

Considerable prior work has been done in guiding the attention of users in VR and MR. We compare the most relevant and recent works in AG research in five categories, which are presented in Table 1.

We are interested in comparing visual AG methods with haptic AG methods to see if the latter can be considered more subtle but still comparable in terms of performance. In particular, Haptic Feedback 
Table 1: Overview on recent work in AG research (TLM: Temporal Luminance Modulation; HF: Haptic Feedback)

\begin{tabular}{|l|c|c|c|c|c|c|c|}
\hline \multirow{2}{*}{ Work } & \multicolumn{3}{|c|}{ Methods } & $\begin{array}{c}\text { Guiding } \\
\text { Attention }\end{array}$ & $\begin{array}{c}\text { Real } \\
\text { Walking }\end{array}$ & $\begin{array}{c}\text { User } \\
\text { Calibrated }\end{array}$ & $\begin{array}{c}\text { Comparative } \\
\text { Study }\end{array}$ \\
\hline & Arrow & TLM & HF & $\checkmark$ & $\checkmark$ \\
\hline HapticHead [8] & & & $\checkmark$ & $\checkmark$ & & & \\
\hline Luminance Modulation [4] & & $\checkmark$ & & $\checkmark$ & & & $\checkmark$ \\
\hline PulseLight and PulseVibe [16] & & $\checkmark$ & $\checkmark$ & $\checkmark$ & & & \\
\hline VRHapticDrones [7] & & & $\checkmark$ & & $\checkmark$ & & \\
\hline FacePush [1] & & & $\checkmark$ & $\checkmark$ & & & \\
\hline GazeRecall [12] & & $\checkmark$ & & $\checkmark$ & & & \\
\hline Visual Stimuli [2] & & $\checkmark$ & & $\checkmark$ & & & \\
\hline Diegetic Cues [13] & & & & $\checkmark$ & & & $\checkmark$ \\
\hline Scaptics [10] & $\checkmark$ & $\checkmark$ & & $\checkmark$ & & $\checkmark$ & \\
\hline Directing vs. Attracting [14] & $\checkmark$ & $\checkmark$ & $\checkmark$ & & \\
\hline Arrow, Butterfly, Radar [18] & $\checkmark$ & & & $\checkmark$ & & & \\
\hline Attention Funnel [17] & $\checkmark$ & & & $\checkmark$ & $\checkmark$ & & $\checkmark$ \\
\hline Rubber Band [15] & $\checkmark$ & & & $\checkmark$ & $\checkmark$ & & $\checkmark$ \\
\hline This work & $\checkmark$ & $\checkmark$ & $\checkmark$ & $\checkmark$ & $\checkmark$ & $\checkmark$ & $\checkmark$ \\
\hline
\end{tabular}

and Temporal Luminance Modulation are generally considered to be AG methods that are not distracting for the user while navigating in a VE $[4,10,12,16]$. Further, they can be better applied together with redirection techniques, as introduced by Razzaque [11]. In order to assess the AG's performance of the aforementioned methods, we are interested in comparing them with one of the currently most effective visual AG methods. According to previous work [5,9,14], the arrow is regarded as most effective. Since we do not use any redirection techniques [11] together with so-called "resets" [20] that also use an arrow to indicate a rotation on spot, we used the arrow as a baseline for the comparative study. First, in the Guiding Attention category, we indicate previous works that have evaluated or developed methods for AG. In fact, some of these were originally implemented for other purposes, such as 3D visualization [10] and levitating haptic feedback proxy [7]. Second, we present a Comparative Study between AG methods based on different sensory channels.

We also added the category User Calibrated to indicate whether the AG methods were calibrated for each individual user. A usertailored calibration is important because it can take physical features (e.g. interpupillary distance) into account that might bias the effectiveness of the AG methods. Finally, we add the category Real Walking to distinguish stationary VEs from real walking enabled ones. So far, to our knowledge there is no comparison of the haptic feedback and temporal luminance modulation subtle AG methods in a real walking enabled VE. Thus, our work provides more insights on the effectiveness of our AG methods in direct comparison to a state-of-the-art visual method (i.e. arrow).

\section{Methodology}

To design and implement our controlled VE, we first identify an adequate task to acquire relevant data for comparing different AG methods. Then, we create two VEs in which the users complete our given task using different AG methods. In a subsequent step, we integrate the following AG methods: $3 \mathrm{D}$ arrow, haptic feedback, temporal luminance modulation, and no AG at all. We then conduct a pilot study using the HTC VIVE Pro Eye HMD, two controllers, and the VIVE Wireless Adapter.

\subsection{Task}

The task is a simple search task which consists of seeking target objects that are hidden in a virtual room. The execution of the search task is defined as a session. A search task session is structured as shown in Fig. 1. Red boxes contain actions that the users need to perform, whereas blue boxes contain responses from the VE. Initial and final states are colored in green. The counter $N$ indicates the number of target objects that were found in a single session. The task pipeline is structured as follows:

1. The users enter the VE, target objects are not yet initialized.

2. To keep track of how many target objects are found in a session, a counter $N$ is used starting at $N=0$.

3. The users activate the platform defined as $h u b$ by pressing a button placed on it.

4. The hub initializes a target object in the VE. Moreover, the hub shows the target object the users should seek.

5. The users seek and grab the found target object.

6. The grabbed target object disappears. The counter is updated $(N=N+1)$.

7. If $N=10$ target objects were found, the session is terminated. Otherwise, the procedure continues with step 3.

Following the current state-of-the-art [2, 14, 18, 19,22], we measure the search time between step 3 and 5 for the data collection. Using the search time for all 10 target objects, we then calculate the mean and the median values. We define the search time for a single object as the time span between activating a target object in the room and the moment when the users grab it.

The hub is a table-like platform that is used for different purposes, as shown in Fig. 2a. First, the hub shows text-based messages to the users (e.g. to let them know when the task is completed). Second, the hub contains an interactive button which has to be pressed by the users to generate a target object in the VE. Once a target object is generated, a non-tangible 3D model of the hidden target object is shown on the hub to instruct the user what to seek (see Fig. 2b). Lastly, the hub ensures constant starting conditions in the VE for each target object. Since users need to press the button on the hub to generate the next target object, they always start the search task from the same position. Having a constant starting condition is crucial for allowing independent measurements for the data collection. Consequently, the search time measured for finding a target object is not biased by different starting conditions. Moreover, the conditions remain the same among users, since the hub's location in the VEs does not change between different sessions. 


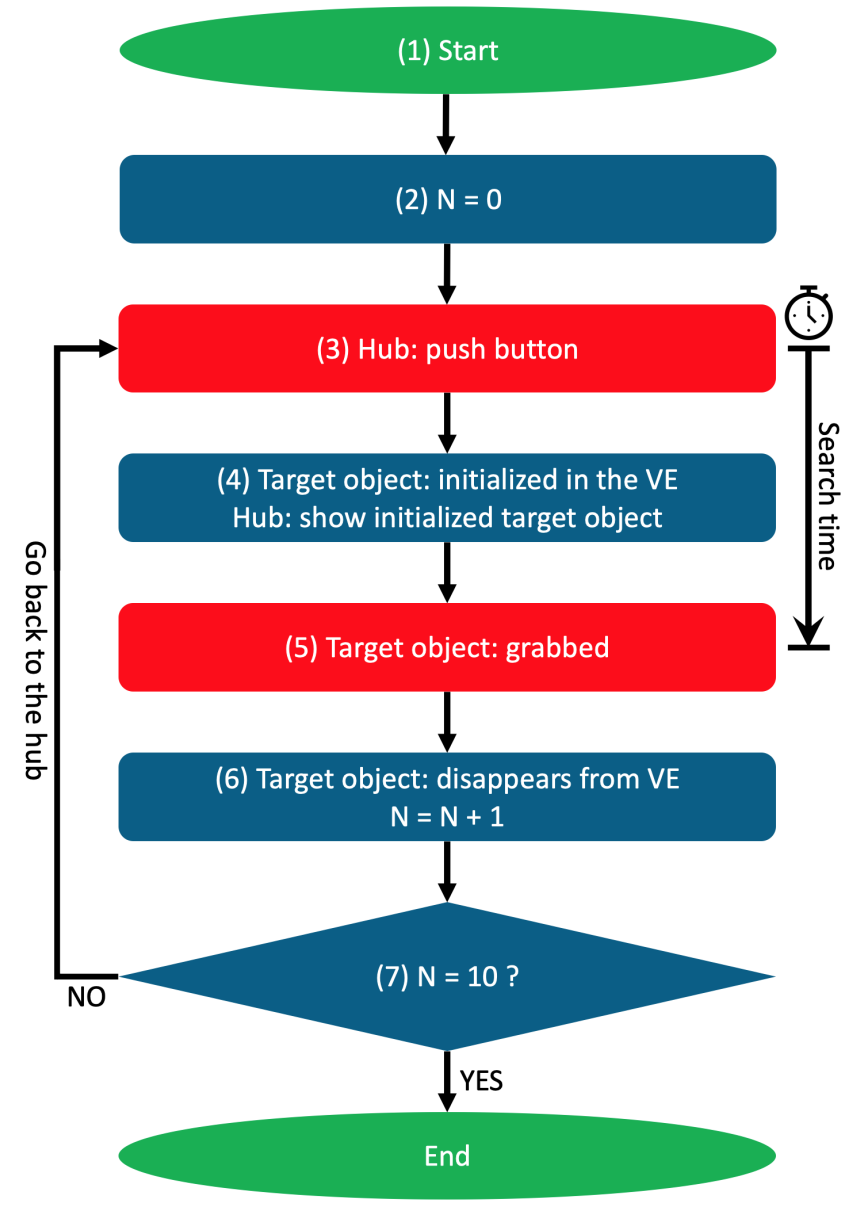

Figure 1: Search task session flowchart. Red boxes indicate actions that users need to perform. Blue boxes indicate responses from the VE. Initial and final states are colored in green. The counter $N$ indicates how many target objects were found in a session. For data collection, the search time is used, measured between step 3 and step 5. Using the search time for all 10 target objects, we then calculate the mean and the median values.

\subsection{Virtual Environments}

Two VEs were designed and implemented: the tutorial VE and the study VE. In both VEs, virtual objects were taken from a common domestic setting (i.e. living-room-style) to reproduce a realistic environment. The tutorial VE is a small VE $(2.5 m \times 4.5 m)$ in which we instruct the user how to engage with the VR hardware (e.g. VR controllers) and interact with the virtual objects (e.g. collision feedback from virtual walls, grabbing virtual objects). Finally, the tutorial VE is also used to teach the user how to complete the searching task session and how to interact with the hub. The study VE is used to conduct the search task and to collect data. Compared to the tutorial VE, the study VE is larger $(4.0 m \times 7.5 m)$ so that users have a larger walking area where they can freely move, which makes the envisioned guidance necessary. Moreover, a large VE makes it possible to create small virtual sectors to hide target objects effectively behind other virtual objects, as shown in Fig. 3. The various target objects are also deliberately placed where they cannot be seen from the hub location, preventing users from finding the target object before starting to walk in the VE. To avoid capturing the user's attention too easily, target objects were taken from the same domestic setting used for

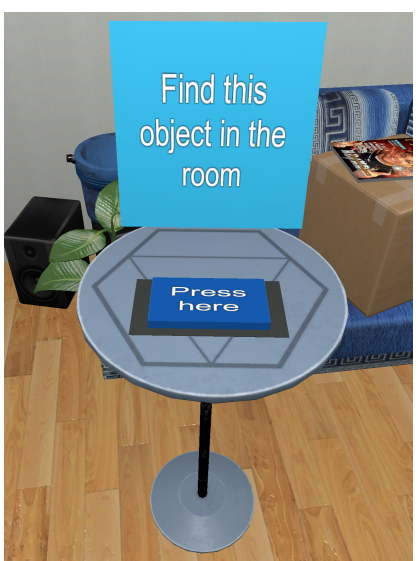

(a) Hub platform appearance.

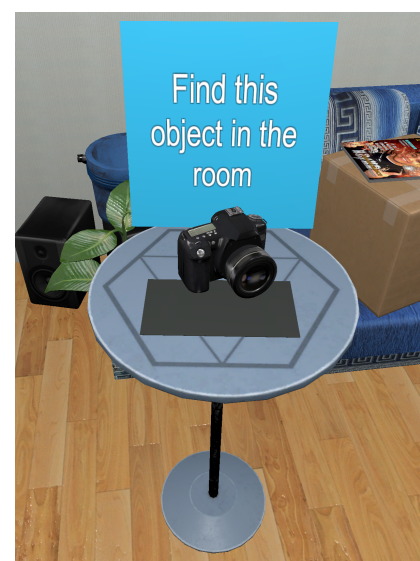

(b) A non-tangible 3D model of the hidden target object (i.e. camera) is shown on the hub.

Figure 2: Hub platform.

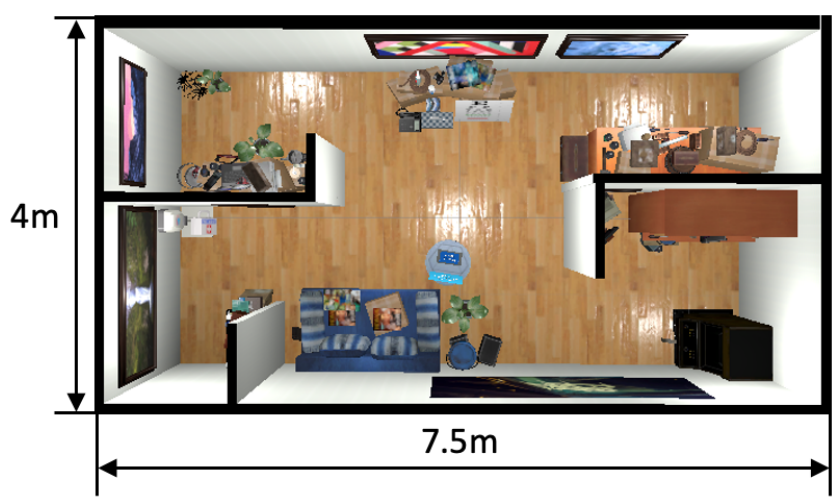

Figure 3: Study environment top-down view.

the VE (e.g. camera, laptop, hairdryer). If users find a target object, they need to grab it with the VR controller by touching the object and pressing a button. In previous work [2, 13, 14, 18,22], usually line of sight trajectories or head direction were used to check if the users have found the target objects. However, this could lead to false positive situations in which the target objects were accidentally in the line of sight of the users (e.g. as a result of an incidental glance), while users might not have consciously noticed these objects. Thus, forcing the users to grab a target object confirms that they have really found it. Moreover, target objects are not all generated at the same time in the VE to avoid any possible learning effects. The next target object is only generated after the previous target object has been found, and the user has returned to the hub and pressed the push button again.

\subsection{Attention Guidance Methods}

In our work, we compared the AG methods: haptic feedback, 3D arrow, and temporal luminance modulation. According to previous work $[4,10,12,16]$, haptic feedback and temporal luminance modulation are more subtle than the $3 \mathrm{D}$ arrow and most likely comparable in terms of AG effectiveness. The AG methods are only active if a target object is generated in the room by pressing the pushbutton on the hub.

First, we adapted the $3 D$ arrow AG method proposed by Schmitz et al. [14] and Wallgrün et al. [18], which consists of a white arrow pointing towards a target object, as shown in Fig. 4a. The arrow 


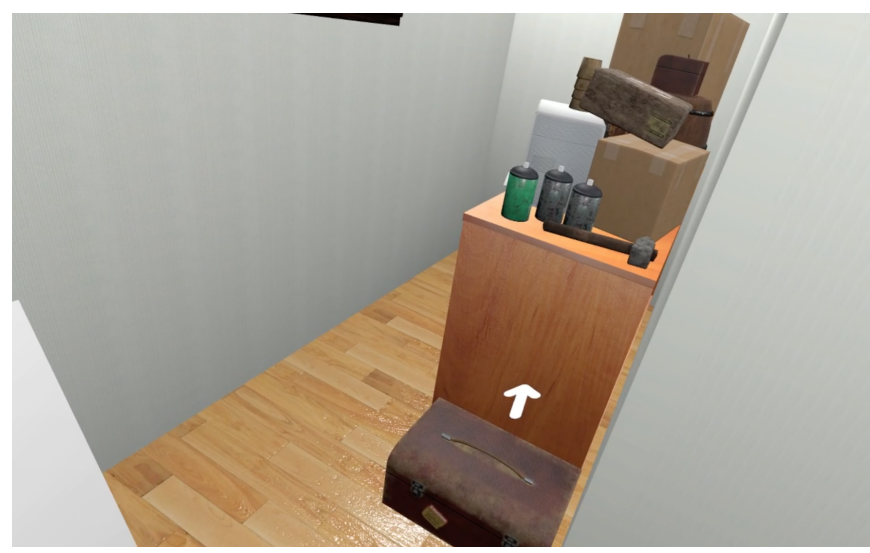

(a) 3D arrow AG method. The arrow is placed in the user's FOV and guides them towards the target object.

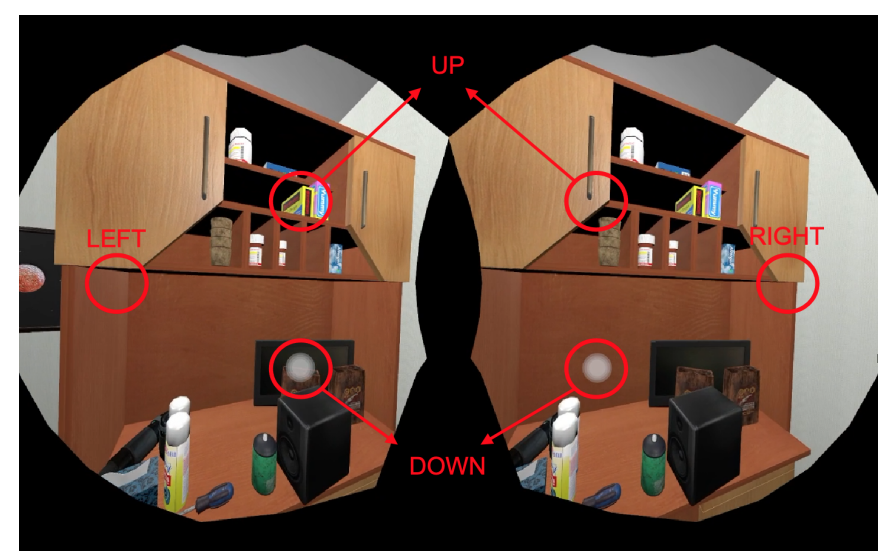

(b) Temporal luminance modulation AG method. For better visualization, a red overlay is added to show the placement in which the flickering artefacts appear. Note that the flickering artefacts in fact appear in a user's peripheral FOV, while the screen capture of the HMD shows them closer to the center of the image.

Figure 4: AG methods that were used in the pilot study.

can rotate around all three axes in space, and moves with the user's head motion while being fixed to the FOV. According to previous work, the 3D arrow is adequate for search tasks as it gives strong and clear guidance [14]. Among visual AG methods, the 3D arrow is usually most preferred by the users as it is intuitive and aesthetically pleasing [18].

Second, we implemented a haptic feedback method proposed by Prouzeau et al. [10] that addresses the tactile sensory channel. In fact, the haptic feedback uses the haptic actuators of both controllers to provide users with information regarding the location of a target object. Only the haptic actuator with the closest proximity to the target object provides vibration feedback, so the user knows in which direction they should rotate. Moreover, both vibration frequency and amplitude are modulated linearly dependent on the distance between the controller and the target object. In particular, the vibration frequency ranges from $20 \mathrm{~Hz}$ to $60 \mathrm{~Hz}$. As a consequence, for users it feels like the intensity of vibration increases if they get closer to the target object.

Temporal luminance modulation is the third and last AG method that we adapted from the one proposed by Schmitz et al. [14], and addresses the visual sensory channel. The temporal luminance modulation consists of four flickering artefacts placed close to the edges of the HMD's screen to guide the attention of the user towards a target object (see Fig. 4b). Because users have different FOVs, the position of these artefacts needs to be adjustable. Thus, we adapted a calibration procedure from Grogorick et al. [4], which is performed by an external operator using the keyboard. Each artefact is built by placing four concentric dots of slightly different diameters and saturation on top of each other to achieve a smoothing effect around the artefact. The flickering effect is generated by making these dots change their color between white and black at $10 \mathrm{~Hz}$. As shown in the work of Schmitz et al. [14], this frequency is low enough to be perceivable by the human eye of every user. In practice, if users are not looking in the direction of the target object, these artefacts appear in their peripheral FOV, encouraging them to turn their head towards the target object. Thus, users can perceive the flickering artefacts, but are not able to recognize them in terms of form and shape.

\subsection{Pilot Study}

We conducted a pilot study with seven participants recruited from local university staff who were not familiar with the AG methods. Participants were instructed that a new target object will become vis- ible at a random position in the room. Moreover, we only informed the participants that they might receive some guidance from the VR system that could help them in finding a target object faster, but we did not provide any further information about the kind of stimuli. Each participant underwent the same study pipeline:

- Training in the tutorial VE.

- Search task session with 3D arrow AG method in the study VE.

- Search task session without any AG in the study VE.

- Search task session with haptic feedback AG method in the study VE.

- Calibration procedure for the temporal luminance modulation AG method in the study VE.

- Search task session with temporal luminance modulation AG method in the study VE.

The placement of the ten target objects was different in each session to avoid any learning effect.

\section{Results and Discussion}

For each user, data was collected from the four search task sessions. For each of these sessions, ten search time measurements were recorded in the VE. However, we removed two search time measurements recorded from different searching task sessions due to technical issues. Consequently, we replaced these two entries with the average search time over all the other users for the same target object and with the same AG method applied. In Fig. 5a, our preliminary results are shown using a box-and-whisker plot. As expected, the results show that the $3 \mathrm{D}$ arrow AG method was the most effective one in terms of search time ( mean $=9.22 \mathrm{~s}$, median $=7.45 \mathrm{~s}$ ), scoring the lowest average and median search time compared to the other AG methods. This is in line with the results presented in literature $[14,18]$. Our preliminary results also show that both - haptic feedback $($ mean $=20.74 s$, median $=13.80$ s $)$ and temporal luminance modulation $($ mean $=11.13 \mathrm{~s}$, median $=9.71 \mathrm{~s})$ - methods are able to reduce the search time with regard to the "no guidance" condition (mean $=33.43$ s, median $=19.39 \mathrm{~s}$ ). Moreover, the temporal luminance modulation method comes closer to the $3 \mathrm{D}$ arrow 


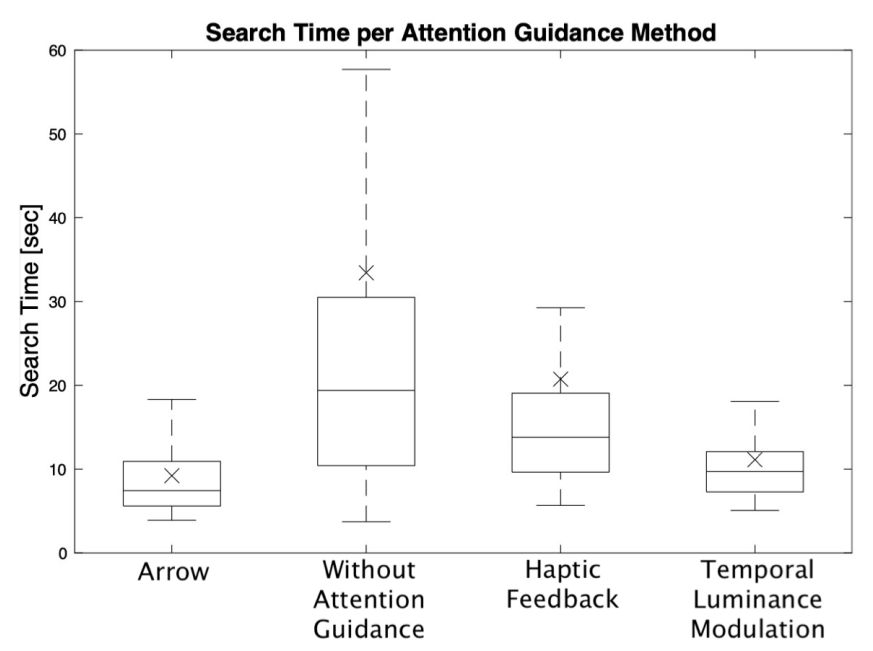

(a) Box-and-whisker plot of the search time per AG method. Mean values are indicated with an " $X$ ".

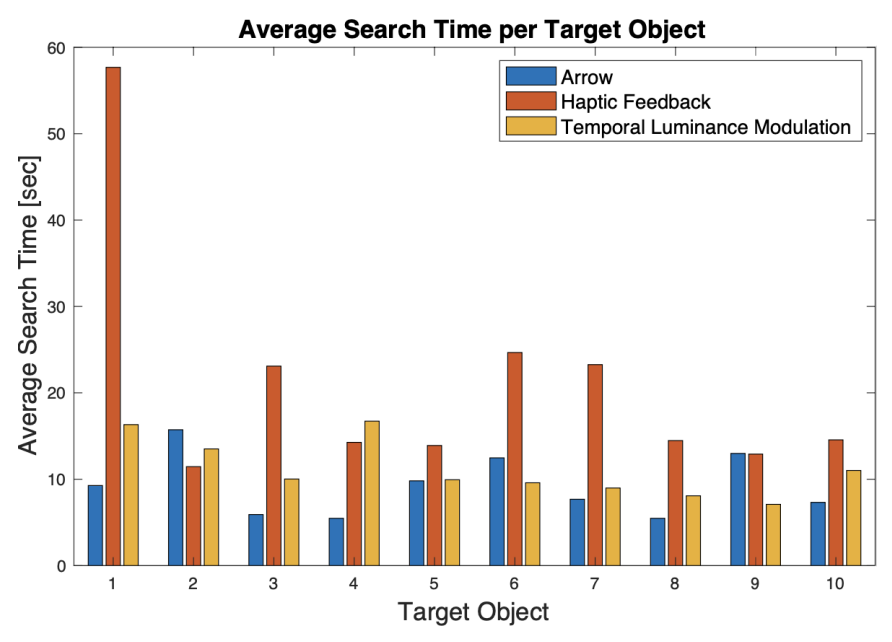

(b) Bar chart of the average search time per target object.

Figure 5: Results of the comparative pilot study.

method in terms of effectiveness, and has the benefit of being less obtrusive.

Fig. 5b shows the average search time per target object for all users. According to the preliminary results, when using the haptic feedback AG method, the search time for finding the first target object is by far greater than the average search time over all the other nine target objects using the same method. Most likely, this originates from the haptic feedback being perceived as less intuitive at first or being mistaken for a collision alert, requiring more time to adapt and interpret the vibration's modulation.

\section{LIMITATIONS}

In our pilot study, we identified different limitations. The haptic feedback may be misinterpreted due to conflicts with the collision feedback, which is also represented by the vibration that users receive from the controllers while colliding with a virtual object. Moreover, compared to the other two AG methods, our haptic feedback implementation is not able to provide upwards and downwards guidance in the VE. Thus, haptic feedback could only be used in open virtual spaces without any obstacles, while it should be discarded in more constraint VEs.

Another general limitation was related to our AG methods' implementation being blind to occlusion. The AG methods led the users towards a target object, but virtual objects between the user's position and the target position were not taken into consideration by the AG methods, i.e. the methods did not guide a user around an obstacle. For example, users could have been led towards a target object, but could not see and reach it because of a virtual wall blocking the way.

\section{Conclusion}

In this paper, we compared three AG methods (see Sect. 3.3) in a controlled VE that enables users to navigate the VE by real walking.

First, we implemented the haptic feedback method, an AG method which uses the tactile sensory channel to guide users towards a target object. This method activates the vibration on the controller that is closer to target object. Moreover, the frequency and amplitude of the vibration are modulated depending on the distance between controller and target object. In this way, users can retrieve information about a target object's location and consequently determine the path to reach it.
Second, we implemented the temporal luminance modulation method, which is a visual AG method that makes use of flickering artefacts placed at the edges of users' peripheral FOV to guide them towards the target objects. These flickering artefacts appear in the direction in which a user should move to reach the target object. To assess the AG methods' effectiveness, we additionally defined two conditions: the no- $A G$ condition and the $3 \mathrm{D}$ arrow $\mathrm{AG}$ method taken from the state-of-the-art. In the pilot study, participants needed to complete a search task in the VE (see Sect. 3.4), while the different AG methods were applied. The obtained preliminary results (see Sect. 4) indicate that the overt 3D arrow AG is the most effective AG method in terms of search time, which is in line with previous work $[14,18]$. The preliminary results further suggest that the temporal luminance modulation compares well to the $3 \mathrm{D}$ arrow AG method in terms of effectiveness (i.e. search time), while being less overt. However, a larger user study will be conducted to reliably assess the performance of these two subtle methods. Since our current AG methods did not take into account obstacles, users could be guided on impossible paths (e.g. through walls) from which they might not see or reach the target object. Thus, our new implementation will integrate possible walking trajectories as proposed by Zank et $a l$. [21] to the target object in the VE as an input for the AG. This can be seen as a more natural and invisible implementation of the "rubber band" approach introduced by Schwerdtfeger et al. [15], where this has been applied in an MR application. Further, other measures than the completion time will be investigated, such as total angle of head rotation, total angle of body rotation, or total length of the walking trajectory. Moreover, to ideally remove any biasing from the study, a new VE should be used for each target object search and the spawn order of target objects should be randomized. To further explore the temporal luminance modulation method in the peripheral FOV as a viable alternative to overt AG methods, future work should also investigate a way to measure the subtleness of the AG methods (e.g. by using an eye tracker). Currently, the flickering artefacts are set manually in terms of size and appearance. Thus, the temporal luminance modulation method might not be suitable for every kind of VE, which might have different brightness levels, different colors, etc. Here, eye tracking and measuring pupil dilation could be used for instance to automatically adapt the appearance of the artefact in terms of position, size, flicker frequency, color, and brightness. This might guarantee an optimal guidance of the user in different regions of a VE. 


\section{REFERENCES}

[1] H.-Y. Chang, W.-J. Tseng, C.-E. Tsai, H.-Y. Chen, R. L. Peiris, and L. Chan. FacePush: Experiencing Pressure Forces on Face with HMDs. In SIGGRAPH Asia 2018 Emerging Technologies, pp. 1-2. ACM, 2018. doi: $10.1145 / 3275476.3275480$

[2] S. Grogorick, G. Albuquerque, and M. Magnor. Comparing Unobtrusive Gaze Guiding Stimuli in Head-Mounted Displays. In 2018 25th IEEE International Conference on Image Processing (ICIP), pp. 2805-2809. IEEE, 2018. doi: 10.1109/ICIP.2018.8451784

[3] S. Grogorick and M. Magnor. Subtle visual attention guidance in VR. In Lecture Notes in Computer Science (including subseries Lecture Notes in Artificial Intelligence and Lecture Notes in Bioinformatics), vol. 11900 LNCS, pp. 272-284. Springer, 2020. doi: 10.1007/978-3 -030-41816-8_11

[4] S. Grogorick, M. Stengel, E. Eisemann, and M. Magnor. Subtle gaze guidance for immersive environments. In Proceedings of the ACM Symposium on Applied Perception, SAP '17, pp. 1-7. ACM, 2017. doi: 10.1145/3119881.3119890

[5] U. Gruenefeld, D. Ennenga, A. E. Ali, W. Heuten, and S. Boll. Eyesee360: Designing a visualization technique for out-of-view objects in head-mounted augmented reality. In Proceedings of the 5th Symposium on Spatial User Interaction, pp. 109-118, 2017. doi: 10.1145/3131277 .3132175

[6] C. Hirt, V. Holzwarth, J. Gisler, J. Schneider, and A. Kunz. Virtual Learning Environment for an Industrial Assembly Task. In 2019 IEEE 9th International Conference on Consumer Electronics (ICCEBerlin), pp. 337-342. IEEE, 2019. doi: 10.1109/ICCE-Berlin47944. 2019.8966169

[7] M. Hoppe, P. Knierim, T. Kosch, M. Funk, L. Futami, S. Schneegass, N. Henze, A. Schmidt, and T. Machulla. VRHapticDrones: Providing Haptics in Virtual Reality through Quadcopters. In Proceedings of the 17th International Conference on Mobile and Ubiquitous Multimedia, pp. 7-18. ACM, 2018. doi: 10.1145/3282894.3282898

[8] O. B. Kaul and M. Rohs. HapticHead: A Spherical Vibrotactile Grid around the Head for 3D Guidance in Virtual and Augmented Reality. In Proceedings of the 2017 CHI Conference on Human Factors in Computing Systems, pp. 3729-3740. ACM, 2017. doi: 10.1145/3025453. 3025684

[9] A. Marquardt, C. Trepkowski, T. D. Eibich, J. Maiero, E. Kruijff, and J. Schöning. Comparing non-visual and visual guidance methods for narrow field of view augmented reality displays. IEEE Transactions on Visualization and Computer Graphics, 26(12):3389-3401, 2020. doi: 10.1109/TVCG. 2020.3023605

[10] A. Prouzeau, M. Cordeil, C. Robin, B. Ens, B. H. Thomas, and T. Dwyer. Scaptics and highlight-planes: Immersive interaction techniques for finding occluded features in 3D scatterplots. In Proceedings of the 2019 CHI Conference on Human Factors in Computing Systems, pp. 1-12, 2019. doi: 10.1145/3290605.3300555

[11] S. Razzaque. Redirected walking. PhD thesis, The University of North Carolina at Chapel Hill, USA, 2005.

[12] S. Rothe, F. Althammer, and M. Khamis. GazeRecall: Using Gaze Direction to Increase Recall of Details in Cinematic Virtual Reality. In
Proceedings of the 17th International Conference on Mobile and Ubiquitous Multimedia, pp. 115-119. ACM, 2018. doi: 10.1145/3282894. 3282903

[13] S. Rothe and H. Hußmann. Guiding the Viewer in Cinematic Virtual Reality by Diegetic Cues. In Lecture Notes in Computer Science (including subseries Lecture Notes in Artificial Intelligence and Lecture Notes in Bioinformatics), vol. 10850 LNCS, pp. 101-117. Springer, 2018. doi: 10.1007/978-3-319-95270-3_7

[14] A. Schmitz, A. MacQuarrie, S. Julier, N. Binetti, and A. Steed. Directing versus Attracting Attention: Exploring the Effectiveness of Central and Peripheral Cues in Panoramic Videos. In 2020 IEEE Conference on Virtual Reality and 3D User Interfaces (VR), pp. 63-72. IEEE, 2020. doi: 10.1109/VR46266.2020.00024

[15] B. Schwerdtfeger, T. Frimor, D. Pustka, and G. Klinker. Mobile information presentation schemes for supra-adaptive logistics applications. In Z. Pan, A. Cheok, M. Haller, R. W. H. Lau, H. Saito, and R. Liang, eds., Advances in Artificial Reality and Tele-Existence, pp. 998-1007. Springer Berlin Heidelberg, Berlin, Heidelberg, 2006. doi: 10.1007/ 11941354_103

[16] T. C. Stratmann, A. Löcken, U. Gruenefeld, W. Heuten, and S. Boll. Exploring Vibrotactile and Peripheral Cues for Spatial Attention Guidance. In PerDis 2018 - Proceedings of the 7th ACM International Symposium on Pervasive Displays, PerDis '18, pp. 1-8. ACM, 2018. doi: 10.1145/3205873.3205874

[17] J. Tümler, R. Mecke, T. Rößler, D. Schenk, and I. Böckelmann. Examining the augmented reality funnel: Does it lead to inattentional blindness? Inter Collegas, 2(3):291-310, 2015. doi: 10.35339/ic.2.3. 291-310

[18] J. O. Wallgrün, M. M. Bagher, P. Sajjadi, and A. Klippel. A Comparison of Visual Attention Guiding Approaches for $360^{\circ}$ Image-Based VR Tours. In 2020 IEEE Conference on Virtual Reality and $3 D$ User Interfaces (VR), pp. 83-91. IEEE, 2020. doi: 10.1109/VR46266.2020. 00026

[19] X. Wang, L. Lin, M. Han, and J. M. Spector. Impacts of cues on learning: Using eye-tracking technologies to examine the functions and designs of added cues in short instructional videos. Computers in Human Behavior, 107:106279, 2020. doi: 10.1016/j.chb.2020.106279

[20] B. Williams, G. Narasimham, B. Rump, T. P. McNamara, T. H. Carr J. Rieser, and B. Bodenheimer. Exploring large virtual environments with an hmd when physical space is limited. In Proceedings of the 4th symposium on Applied perception in graphics and visualization, pp. 41-48, 2007. doi: 10.1145/1272582.1272590

[21] M. Zank and A. Kunz. Optimized graph extraction and locomotion prediction for redirected walking. In M. M. R. J. T. B. Thomas, ed., IEEE Symposium on 3D User Interfaces, pp. 120-129. IEEE, Washington, USA, 2017. doi: 10.1109/3DUI.2017.7893328

[22] J. Zhang, M. Mullikin, Y. Li, and C. Mei. A Methodology of Eye Gazing Attention Determination for VR Training. In 2020 IEEE Conference on Virtual Reality and 3D User Interfaces Abstracts and Workshops (VRW), pp. 138-141. IEEE, 2020. doi: 10.1109/VRW50115. 2020.00029 